\title{
STRONG UNIQUE CONTINUATION FOR THE LAMÉ SYSTEM WITH LIPSCHITZ COEFFICIENTS IN THREE DIMENSIONS*
}

\author{
HANG $\mathrm{YU}^{1}$
}

\begin{abstract}
This paper studies the strong unique continuation property for the Lamé system of elasticity with variable Lamé coefficients $\lambda, \mu$ in three dimensions, $\operatorname{div}\left(\mu\left(\nabla u+\nabla u^{t}\right)\right)+\nabla(\lambda \operatorname{div} u)+V u=0$ where $\lambda$ and $\mu$ are Lipschitz continuous and $V \in L^{\infty}$. The method is based on the Carleman estimate with polynomial weights for the Lamé operator.
\end{abstract}

Mathematics Subject Classification. 35B60, 74B05.

Received September 24, 2009. Revised January 25, 2010.

Published online April 23, 2010.

\section{INTRODUCTION}

The main purpose of this paper is to study the strong unique continuation for the Lamé system with $C^{0,1}$ coefficients in three dimensions. Firstly, let us introduce the Lamé system of elasticity. Assume that $\Omega$ is a bounded domain in $\mathbb{R}^{3}$, the Lamé moduli $\mu=\mu(x), \lambda=\lambda(x)$ are $C^{0,1}(\Omega)$ and satisfy the strong ellipticity conditions

and the upper bound

$$
\mu \geq \alpha_{0}>0, \quad 2 \mu+\lambda \geq \beta_{0}>0,
$$

$$
\|\mu\|_{C^{0,1}(\Omega)}+\|\lambda\|_{C^{0,1}(\Omega)} \leq C
$$

where $\alpha_{0}, \beta_{0}$ and $C$ are given positive constants. Without loss of generality, we may assume that $\Omega$ contains the origin and $B_{R} \subset \subset \Omega$ for some $R>0$ where $B_{R}$ is the open ball centered at the origin with radius $R$. The Lamé system with a perturbation $V \in L^{\infty}(\Omega)$ is given by

$$
\operatorname{div}\left(\mu\left(\nabla u+(\nabla u)^{\top}\right)\right)+\nabla(\lambda \operatorname{div} u)+V u=0,
$$

where $u=\left(u_{1}, u_{2}, u_{3}\right)^{\top}$ is the displacement vector and

$$
\nabla u=\left(\begin{array}{ccc}
\partial_{x_{1}} u_{1} & \partial_{x_{2}} u_{1} & \partial_{x_{3}} u_{1} \\
\partial_{x_{1}} u_{2} & \partial_{x_{2}} u_{2} & \partial_{x_{3}} u_{2} \\
\partial_{x_{1}} u_{3} & \partial_{x_{2}} u_{3} & \partial_{x_{3}} u_{3}
\end{array}\right)
$$

is the gradient matrix of $u$.

\footnotetext{
Keywords and phrases. Lamé system, Carleman estimate, strong unique continuation.

* The author was supported in part by NSFC (No. 10801041 and 10831007), FANEDD (No. 200522) and NCET (No. 06-0359).

${ }^{1}$ School of Mathematical Sciences, Fudan University, 200433 Shanghai, P.R. China. hangyumath@hotmail.com
} 
We recall that a function $u \in L_{\text {loc }}^{2}\left(\mathbb{R}^{n}\right)$ is said to vanish of infinite order at a point $x_{0}$ if for any $k>0$,

$$
\int_{\left|x-x_{0}\right|<r}|u|^{2} \mathrm{~d} x=O\left(r^{k}\right), \quad \text { as } r \rightarrow 0 .
$$

We say that (1.1) has the strong unique continuation property (SUCP) if any solution $u$ is identically zero whenever it vanishes of infinite order at a point of $\Omega$.

The previous results in the literature are the following. The result of the (weak) unique continuation for the Lamé system was first given by Dehman and Robbiano for $\lambda, \mu \in C^{\infty}\left(\mathbb{R}^{n}\right)$ [3]. They proved the Carleman estimate by pseudodifferential calculus. Then Ang et al. gave a result for $\lambda \in C^{2}\left(\mathbb{R}^{n}\right), \mu \in C^{3}\left(\mathbb{R}^{n}\right)[2]$; Weck proved a result for $\lambda, \mu \in C^{2}\left(\mathbb{R}^{n}\right)[12,13]$. On the other hand, the result on the strong unique continuation (SUCP) for the Lamé system was first obtained by Alessandrini and Morassi for $n \geq 2, \lambda, \mu \in C^{1,1}\left(\mathbb{R}^{n}\right)$ [1]. Then Lin and Wang studied the SUCP in the case of $n=2, \lambda, \mu \in W^{1, \infty}\left(\mathbb{R}^{n}\right)$ [8]. Their proof relies on reducing the Lamé system to a first order elliptic system and on some suitable Carleman estimates with polynomial weights. Later, the result of [8] was improved to $\mu \in C^{0,1}(\Omega)$ and $\lambda$ being measurable by Escauriaza [5]. Also, while submitting this article, we became aware of the work of Lin et al. [7] which also consider SUCP for the Lamé operator with Lipschitz coefficients.

The purpose of this paper is to consider the more general case in (1.1). Our result is as follows:

Theorem 1.1. Assume that $\Omega$ is a bounded domain in $\mathbb{R}^{3}$, the Lamé coefficients $\mu, \lambda \in C^{0,1}(\Omega)$ satisfy the strong elliptic conditions. Let $u \in H^{2}(\Omega)$ be a solution to (1.1). If there is a point $x_{0} \in \Omega$ such that, for every $k \in \mathbb{N}$,

then $u \equiv 0$, in $\Omega$.

$$
\int_{\left|x-x_{0}\right|<r}|u|^{2} \mathrm{~d} x=O\left(r^{k}\right), \quad \text { as } r \rightarrow 0,
$$

As we shall see in Section 3, Theorem 1.1 is a combination of two results: first, we will show that if $u$ solves (1.1), then $u$ cannot have zeros of "exponential" order, i.e., there is no point $x_{0} \in \Omega$ such that

$$
\int_{\left|x-x_{0}\right|<r}|u|^{2} \mathrm{~d} x=O\left(\mathrm{e}^{-r^{-1}}\right), \quad \text { as } r \rightarrow 0,
$$

unless $u$ is identically 0 . This result can be seen in our previous work [14]. Next, we will prove if $x_{0}$ is a zero of infinite order for $u$, then $x_{0}$ is a zero of "exponential" order, i.e. (1.2) implies (1.3). For this step we need the Carleman estimates with polynomial weights.

\section{Carleman estimates with polynomial Weights}

In [11], the authors give a result of the Carleman estimates for Dirac operators. The proof uses the fact that Dirac squared is the Laplacian. Inspired by $[6,11]$, one can find a way to obtain the Carleman estimates with polynomial weights for Lamé operator directly from a corresponding estimates for the Laplacian in three dimensions, since the Lamé operator is a composition of two first order operators, one of which is a Dirac operator. For doing this, we need to use the semiclassical Sobolev spaces

$$
\|u\|_{H_{s c l}^{s}}=\left\|\langle h D\rangle^{s} u\right\|_{L^{2}}
$$

where $\langle\xi\rangle=\left(1+|\xi|^{2}\right)^{\frac{1}{2}}$. It is easy to prove that if $s$ is a positive integer, then

$$
\|u\|_{H_{s c l}^{s}} \approx \sum_{0 \leq k \leq s}\left(\left\|h^{k} D^{k} u\right\|_{L^{2}}\right) .
$$

Here and below $\|\cdot\|=\|\cdot\|_{L^{2}\left(\mathbb{R}^{n}\right)}$ and $C$ denoting a constant independent of $h$ and $u$, may vary from line to line. 
Firstly, we give a generalized Carleman estimate with polynomial weights for the Laplacian. Below, we will use the conventions of semiclassical calculus. In particular we consider the usual semiclassical symbol classes $S^{m}$, and relate to symbols $a \in S^{m}$ operators $A=\mathrm{Op}_{h}(a)$ via Weyl quantization. See [9] for more details.

Lemma 2.1. Let $0 \leq s \leq 1$. Then for any $u \in C_{c}^{\infty}\left(B_{r} \backslash\{0\}\right)$ where $B_{r} \subset \mathbb{R}^{n}$ is a ball of radius $r$ around the origin 0 and for any $\frac{1}{h}=k+\frac{n+1}{2}, k \in \mathbb{N}$, we have the estimate

$$
\left\||x|^{-\frac{1}{h}} u\right\|_{H_{s c l}^{s+1}} \leq C h r^{2}\left\||x|^{-\frac{1}{h}} \Delta u\right\|_{H_{s c l}^{s-1}}
$$

where $C$ depends only on $n$.

Proof. Consider the Carleman inequality for the Laplace operator proved by Regbaoui [10],

$$
\int|x|^{-\frac{2}{h}-4}|u|^{2} \mathrm{~d} x+h^{2} \int|x|^{-\frac{2}{h}-2}|D u|^{2} \mathrm{~d} x+h^{4} \int|x|^{-\frac{2}{h}}\left|D^{2} u\right|^{2} \mathrm{~d} x \leq C h^{2} \int|x|^{-\frac{2}{h}}|\Delta u|^{2} \mathrm{~d} x
$$

for all $u \in C_{c}^{\infty}\left(B_{r} \backslash\{0\}\right)$, where $\frac{1}{h}=k+\frac{n+1}{2}, k \in \mathbb{N}$.

Setting $t=h r$, we define

$$
\|u\|_{H_{s c l}^{s}}=\left\|\langle t D\rangle^{s} u\right\|_{L^{2}}
$$

where $\langle\xi\rangle=\left(1+|\xi|^{2}\right)^{\frac{1}{2}}$. Let

and

$$
P=|x|^{-\frac{1}{\hbar}} \circ\left(-t^{2} \Delta\right) \circ|x|^{\frac{1}{\hbar}}
$$

$$
u=|x|^{\frac{1}{h}} v \in C_{c}^{\infty}\left(B_{r} \backslash\{0\}\right) .
$$

Putting $u$ into (2.2), one has

$$
\begin{aligned}
I_{1} & =\left\||x|^{-2} v\right\|+h\left\||x|^{-\frac{1}{h}-1} D\left(|x|^{\frac{1}{h}} v\right)\right\|+h^{2}\left\||x|^{-\frac{1}{h}} D^{2}\left(|x|^{\frac{1}{h}} v\right)\right\| \\
& \leq C h\left\||x|^{-\frac{1}{h}} \Delta\left(|x|^{\frac{1}{h}} v\right)\right\|=C t^{-1} r^{-1}\|P v\|=I_{2} .
\end{aligned}
$$

Let $0<\eta \ll \varepsilon \ll 1$.

$$
\begin{aligned}
I_{1} & \geq\left\||x|^{-2} v\right\|+\varepsilon h\left\||x|^{-1}|D v|+\frac{1}{h}|x|^{-2}(D|x|) v\right\|+\eta h^{2}\left\|D^{2} v+|x|^{-\frac{1}{h}}\left[D^{2},|x|^{\frac{1}{h}}\right] v\right\| \\
& \geq\left\||x|^{-2} v\right\|+\varepsilon h\left\||x|^{-1}|D v|+\frac{1}{h}|x|^{-2}(D|x|) v\right\|+\eta h^{2}\left\|D^{2} v+\frac{1}{h}|x|^{-1} D(|x|) D v+\frac{1}{h}\left(\frac{1}{h}-1\right)|x|^{-2} v\right\| \\
& \geq\left\||x|^{-2} v\right\|+\varepsilon\left(h\left\||x|^{-1}|D v|\right\|-C\left\||x|^{-2} v\right\|\right)+\eta\left(h^{2}\left\|D^{2} v\right\|-h C\left\||x|^{-1} D v\right\|-C\left\|\left.|| x\right|^{-2} v\right\|\right) \\
& \geq(1-C \varepsilon-C \eta)\left\||x|^{-2} v\right\|+h(\varepsilon-C \eta)\left\||x|^{-1} D v\right\|+h^{2}\left\|D^{2} v\right\| \\
& \geq C\left(\left\||x|^{-2} v\right\|+h\left\||x|^{-1} D v\right\|+h^{2}\left\|D^{2} v\right\|\right) \\
& \geq C\left(r^{-2}\|v\|+h r^{-1}\|D v\|+h^{2}\left\|D^{2} v\right\|\right) .
\end{aligned}
$$

Composing (2.3) and (2.4), we rewrite (2.2) as follows

$$
\|v\|+r h\|D v\|+r^{2} h^{2}\left\|D^{2} v\right\| \leq C r t^{-1}\|P v\| .
$$


Then we have

$$
\|v\|_{H_{s c l}^{2}} \leq C r t^{-1}\|P v\|, \text { for all } v \in C_{c}^{\infty}\left(B_{r} \backslash\{0\}\right) .
$$

Suppose $v \in C_{c}^{\infty}\left(B_{r} \backslash\{0\}\right)$, then $\langle t D\rangle^{j} v \in \mathscr{S}, \forall j \in \mathbb{R}$. In order to use the Carleman estimate we have to cut $\langle t D\rangle^{j} v$ off.

Let $\chi \in C_{c}^{\infty}\left(B_{2 r} \backslash\{0\}\right)$ such that

$$
(1-\chi) v=0
$$

Note that $\operatorname{supp}(1-\chi) \cap \operatorname{supp} v=\emptyset$, and this yields the estimate

$$
\left\|(1-\chi)\langle t D\rangle^{j} v\right\|_{H_{s c l}^{l}} \leq C_{M} t^{M}\|v\|_{H_{s c l}^{k}}, \text { for any } l, k, j, M .
$$

This and (2.6) imply

$$
\begin{aligned}
\|v\|_{H_{s c l}^{s+1}} & =\left\|\langle t D\rangle^{s-1} v\right\|_{H_{s c l}^{2}} \\
& \leq\left\|\chi\langle t D\rangle^{s-1} v\right\|_{H_{s c l}^{2}}+\left\|(1-\chi)\langle t D\rangle^{s-1} v\right\|_{H_{s c l}^{2}} \\
& \leq C\left(r t^{-1}\left\|P\left(\chi\langle t D\rangle^{s-1} v\right)\right\|+t\|v\|_{H_{s c l}^{s+1}}\right) \\
& \leq C t^{-1}\left(r\left\|\chi\langle t D\rangle^{s-1} P v\right\|+r\left\|\chi\left[P,\langle t D\rangle^{s-1}\right] v\right\|+r\left\|[P, \chi]\langle t D\rangle^{s-1} v\right\|+t^{2}\|v\|_{H_{s c l}^{s+1}}\right)
\end{aligned}
$$

By the property (2.7) we have

$$
\left\|[P, \chi]\langle t D\rangle^{s-1} v\right\| \leq C_{M} t^{M}\|v\|_{H_{s c l}^{s+1}}, \text { for any } M .
$$

Noting that $|\xi|^{2}$ and $\langle t|\xi|\rangle^{s-1}$ are main symbols of differential operator $P$ and pseudo differential operator $\langle t D\rangle^{s-1}$ respectively, we have $\chi\left[P,\langle t D\rangle^{s-1}\right] \in t \mathrm{Op}_{t}\left(S^{s-1}\right)$, since $\left\{|\xi|^{2},\langle t|\xi|\rangle^{s-1}\right\}$ vanishes identically, where $\{a, b\}$ denotes the Poisson bracket of $a$ and $b$, defined by

$$
\{a, b\}=\frac{\partial a}{\partial \xi} \frac{\partial b}{\partial x}-\frac{\partial a}{\partial x} \frac{\partial b}{\partial \xi} .
$$

Hence

$$
\left\|\chi\left[P,\langle t D\rangle^{s-1}\right] v\right\| \leq C t\left\|\langle t D\rangle^{s-1} v\right\|
$$

where $C$ depends only on the dimension. Then by (2.8), (2.9), (2.10) and choosing $0<t \ll r \ll 1$, we have

$$
t\|v\|_{H_{s c l}^{s+1}} \leq C r\|P v\|_{H_{s c l}^{s-1}}
$$

Noting that $v=|x|^{-\frac{1}{h}} u$, we conclude $(2.1)$.

From [4] we know that in three dimensions the Lamé operator $L$ can be written as a composition of two first order operators. Indeed, consider the elliptic operator $A: H^{1}\left(\Omega ; \mathbb{R}^{4}\right) \rightarrow L^{2}\left(\Omega ; \mathbb{R}^{4}\right)$ defined by

$$
A(\partial)(u, v)=(\nabla \times u+\nabla v,-\nabla \cdot u)
$$

and its perturbation $A_{\alpha}: H^{1}\left(\Omega ; \mathbb{R}^{4}\right) \rightarrow L^{2}\left(\Omega ; \mathbb{R}^{4}\right)$ defined by

$$
A_{\alpha}(x, \partial)(u, v)=(\nabla \times u+\alpha \nabla v,-\nabla \cdot u),
$$


where $u$ is a vector-valued function with three components, $v$ and $\alpha$ are scalar-valued functions. $A(\partial)$ is a Dirac operator since $A^{2}=-\Delta I_{4}$. We refer [11] for Carleman estimates for general Dirac operators.

Now we turn to the Lamé system (1.1). Assume $u: \Omega \subset \mathbb{R}^{3} \rightarrow \mathbb{R}^{3}$ denoting the displacement vector. Let $L$ be the Lamé operator

$$
L u:=\mu \Delta u+(\lambda+\mu) \nabla \operatorname{div} u .
$$

It is easy to check that

$$
L u:=\mu \Delta u+(\lambda+\mu) \nabla \operatorname{div} u=(2 \mu+\lambda) \nabla \nabla \cdot u-\mu \nabla \times \nabla \times u .
$$

Then we have

$$
(L u, 0)=(\mu \Delta u+(\lambda+\mu) \nabla \operatorname{div} u, 0)=-\mu A_{\alpha}(x, \partial) A(\partial)(u, 0),
$$

where we choose $\alpha=\frac{(2 \mu+\lambda)}{\mu}$.

Firstly we give the Carleman estimates for $A$ and $A_{\alpha}$ by using our generalized Carleman estimate for Laplace operator.

Lemma 2.2. For any $v \in C_{c}^{\infty}\left(B_{r} \backslash\{0\} ; \mathbb{R}^{4}\right)$ where $B_{r} \subset \mathbb{R}^{3}$ is a ball of radius $r$ around the origin 0 and for any $\frac{1}{h}=k+\frac{n+1}{2}, k \in \mathbb{N}$, we have the estimates

$$
\begin{gathered}
\|v\|_{H_{s c l}^{1}} \leq C_{1} r\left\|\left.|| x\right|^{-\frac{1}{h}} A\left(|x|^{\frac{1}{h}} u\right)\right\|, \\
\|v\|_{H_{s c l}^{1}} \leq C_{2} r\left\||x|^{-\frac{1}{h}} A_{\alpha}\left(|x|^{\frac{1}{h}} v\right)\right\|,
\end{gathered}
$$

where $C_{1}$ and $C_{2}$ are two positive constants depending only on $\alpha$.

Proof. Let

Then we have

$$
\widehat{A}=|x|^{-\frac{1}{h}} \circ(t A) \circ|x|^{\frac{1}{h}} .
$$

$$
\widehat{A}^{2}=|x|^{-\frac{1}{h}} \circ\left(-t^{2} \Delta I_{4}\right) \circ|x|^{\frac{1}{h}} .
$$

We recall the composition formula for semiclassical symbols $p$ and $q$ : the operator $P Q$ has symbol

$$
\sigma(P Q) \sim \sum_{\alpha, \beta} \frac{h^{|\alpha+\beta|}(-1)^{|\alpha|}}{(2 i)^{|\alpha|} \alpha ! \beta !}\left(\partial_{x}^{\alpha} \partial_{\xi}^{\beta} p(x, \xi)\right)\left(\partial_{\xi}^{\alpha} \partial_{x}^{\beta} q(x, \xi)\right) .
$$

Denoting $b(x, \xi)$ the symbol of $\langle h D\rangle^{-1} \widehat{A}$, we have by (2.14)

$$
b(x, \xi) \sim \sum_{\alpha, \beta} \frac{h^{|\alpha|}}{(i)^{|\alpha|}|\alpha| !}\left(\partial_{\xi}^{\alpha}\left(1+|h \xi|^{2}\right)^{-\frac{1}{2}}\right)\left(\partial_{x}^{\alpha} a_{r}(x, \xi)\right)=1+\mathcal{O}(h), \quad \text { in } S^{0} .
$$

Now Lemma 2.1 implies, for any $v \in C_{c}^{\infty}\left(B_{r} \backslash\{0\} ; \mathbb{R}^{4}\right)$

$$
\begin{aligned}
t\|v\|_{H_{s c l}^{1}} & \leq C r\left\||x|^{-\frac{1}{h}} \circ\left(-t^{2} \Delta\right) \circ|x|^{\frac{1}{h}} v\right\|_{H_{s c l}^{-1}} \\
& =C r\left\|\widehat{A}^{2} v\right\|_{H_{s c l}^{-1}} \\
& =C r\left\|\langle t D\rangle^{-1} \widehat{A} \widehat{A} v\right\| \\
& \leq C r\|\widehat{A} v\|
\end{aligned}
$$

since $\langle h r D\rangle^{-1} \widehat{A}$ is of order 0 . 
Substituting $|x|^{-\frac{1}{h}} v$ for $v$ in $(2.15)$, we have

$$
\left\||x|^{-\frac{1}{h}} v\right\|_{H_{s c l}^{1}} \leq C r\left\||x|^{-\frac{1}{h}} A v\right\|
$$

By regularizing, we know (2.16) is valid if we suppose $v \in H^{1}\left(\mathbb{R}^{3}\right)$ with compact support in $B_{r} \backslash\{0\}$ that is a three dimensional ball of radius $r$ centered at 0 but removing the center 0 .

Next, with

$$
I_{\alpha}=\left(\begin{array}{cccc}
1 & 0 & 0 & 0 \\
0 & 1 & 0 & 0 \\
0 & 0 & 1 & 0 \\
0 & 0 & 0 & \alpha
\end{array}\right)
$$

we have

$$
A_{\alpha}(x, \partial)=A(\partial) I_{\alpha}
$$

Noting that $C^{0,1}(\Omega)$ is equivalent to $W^{1, \infty}(\Omega)$, we have

$$
\alpha=\frac{(2 \mu+\lambda)}{\mu} \in W^{1, \infty}(\Omega) .
$$

Thus $\widetilde{v} \in H^{1}\left(\mathbb{R}^{3}\right)$ has compact support in $B_{r} \backslash\{0\}$. Hence by putting $\widetilde{v}$ into $(2.16)$, we have

$$
\begin{aligned}
\left\||x|^{-\frac{1}{h}} I_{\alpha} v\right\|_{H_{s c l}^{1}} & \leq C r\left\||x|^{-\frac{1}{h}} A\left(I_{\alpha} v\right)\right\| \\
& \leq C r\left\||x|^{-\frac{1}{h}} A_{\alpha} v\right\|+C r\left\||x|^{-\frac{1}{h}}\left(A I_{\alpha}\right) v\right\| \\
& \leq C r\left\||x|^{-\frac{1}{h}} A_{\alpha} v\right\|+C_{\alpha} r\left\||x|^{-\frac{1}{h}} v\right\|,
\end{aligned}
$$

where $C_{\alpha}$ is a constant depending on $\alpha$. Noting that the second term of the right hand side can be absorbed by the left hand side, we have (2.13).

The Carleman estimate for the Lamé operator follows immediately by (2.11), (2.12) and (2.13). Indeed, noticing that $|x|^{-\frac{1}{h}} \circ A \circ|x|^{\frac{1}{h}}$ is a differential operator, we have

$$
|x|^{-\frac{1}{h}} A\left(|x|^{\frac{1}{h}} v\right) \in C_{c}^{\infty}\left(\mathbb{R}^{3} \backslash\{0\} ; \mathbb{R}^{4}\right)
$$

for any $u \in C_{c}^{\infty}\left(\mathbb{R}^{3} \backslash\{0\} ; \mathbb{R}^{3}\right)$, let

$$
v=(u, 0) \in C_{c}^{\infty}\left(\mathbb{R}^{3} \backslash\{0\} ; \mathbb{R}^{4}\right),
$$

we have

$$
\begin{aligned}
\left\||x|^{-\frac{1}{h}} A\left(|x|^{\frac{1}{h}} v\right)\right\| & \leq\left\||x|^{-\frac{1}{h}} A\left(|x|^{\frac{1}{h}} v\right)\right\|_{H_{s c l}^{1}} \\
& \leq C r\left\||x|^{-\frac{1}{h}} A_{\alpha} A\left(|x|^{\frac{1}{h}} v\right)\right\| \\
& =C r\left\||x|^{-\frac{1}{h}} L\left(|x|^{\frac{1}{h}} u\right)\right\| .
\end{aligned}
$$

With (2.12) and (2.17), we have a key theorem as follows.

Theorem 2.1. There exists $C$ depending only on $n$ and Lamé coefficients such that for any $u \in C_{c}^{\infty}\left(B_{r} \backslash\{0\}\right)$ where $B_{r} \subset \mathbb{R}^{3}$ is a ball of radius $r$ around the origin 0 and for any $\frac{1}{h}=k+\frac{n+1}{2}, k \in \mathbb{N}$, we have the estimate

$$
\|u\|+r h\|D u\| \leq C r^{2}\left\||x|^{-\frac{1}{h}} L\left(|x|^{\frac{1}{h}} u\right)\right\| .
$$


Remark 2.1. The estimate (2.18) remains valid if we suppose $u \in H_{\mathrm{loc}}^{2}\left(\mathbb{R}^{3}\right)$ with compact support and satisfying for all $|\alpha| \leq 2$ and all $N>0, \int_{B_{r}}\left|D^{\alpha} u\right|^{2} \mathrm{~d} x=O\left(r^{N}\right)$ as $r \rightarrow 0$. We can easily see this by cutting $u$ off for small $r$ and regularizing.

\section{Proof of the MAIN ReSUlt}

We need the following auxiliary lemma.

Lemma 3.1. Suppose $u \in H_{\mathrm{loc}}^{2}\left(\mathbb{R}^{3}\right)$ and $u$ vanishes of infinite order at $x_{0}$. Then $|x|^{-\frac{1}{h}} u$ also vanishes of infinite order at $x_{0}$.

Proof. $u$ vanishes of infinite order at $x_{0}$, then

$$
\int_{B_{r}}|u|^{2} \mathrm{~d} x=O\left(r^{k}\right), \quad \forall k>0 \quad r \rightarrow 0 .
$$

We have

$$
\begin{aligned}
\int_{B_{r}}|x|^{-\frac{2}{h}}|u|^{2} \mathrm{~d} x & =\sum_{n \geq 0} \int_{B_{\frac{r}{2^{n}}} \backslash B \frac{r}{2^{n+1}}}|x|^{-\frac{2}{h}}|u|^{2} \mathrm{~d} x \\
& \leq \sum_{n \geq 0}\left(\frac{r}{2^{n+1}}\right)^{-\frac{2}{h}}\left(\frac{r}{2^{n}}\right)^{k} \\
& =r^{-\frac{2}{h}+k} \sum_{n \geq 0} \frac{1}{2^{n k-(n+1) \frac{2}{h}}} \\
& \leq r^{-\frac{2}{h}+k} .
\end{aligned}
$$

That is

$$
\int_{B_{r}}|x|^{-\frac{2}{h}}|u|^{2} \mathrm{~d} x=O\left(r^{k-\frac{2}{h}}\right), \quad \forall k>0 \text { as } r \rightarrow 0 .
$$

This is the conclusion of the lemma.

By Lemma 3.1, we have another version of Theorem 2.1.

Corollary 3.1. Assume $u \in C_{c}^{\infty}\left(B_{r} \backslash\{0\}\right)$ and $u$ vanishes of infinite order at $x_{0}$. There exists $C$ depending on the Lamé coefficients, such that for all $\frac{1}{h}=k+\frac{n+1}{2}, k \in \mathbb{N}$, it holds

$$
\left\||x|^{-\frac{1}{h}} u\right\|+r h\left\|\left.|| x\right|^{-\frac{1}{h}} D u\right\| \leq C r^{2}\left\||x|^{-\frac{1}{h}} L u\right\| .
$$

Proof. We need only to prove

$$
\left\||x|^{-\frac{1}{h}} u\right\|+r h\left\|D\left(|x|^{-\frac{1}{h}} u\right)\right\| \geq C\left(\left\||x|^{-\frac{1}{h}} u\right\|+r h\left\|\left.|| x\right|^{-\frac{1}{h}} D u\right\|\right) .
$$

Indeed, there exists $c>1$, such that

$$
\begin{aligned}
\left\||x|^{-\frac{1}{h}} u\right\|^{2}+r^{2} h^{2}\left\|D\left(|x|^{-\frac{1}{h}} u\right)\right\|^{2} & \geq\left\||x|^{-\frac{1}{h}} u\right\|^{2}+\left(1-\frac{1}{c}\right) r^{2} h^{2}\left\||x|^{-\frac{1}{h}} D u\right\|^{2}+(1-c) r^{2}\left\||x|^{-\frac{1}{h}-1} u\right\|^{2} \\
& =\left\||x|^{-\frac{1}{h}} u\right\|^{2}+(1-c) r^{2}\left\||x|^{-\frac{1}{h}-1} u\right\|^{2}+\left(1-\frac{1}{c}\right) r^{2} h^{2}\left\||x|^{-\frac{1}{h}} D u\right\|^{2} .
\end{aligned}
$$


Noting that $u$ vanishes of infinite order at $x_{0}$, we set $w=|x|^{-\frac{1}{h}} u$. Then by the proof of Lemma 3.1, we know

$$
\int_{B_{r}}|w|^{2} \mathrm{~d} x=O\left(r^{k-\frac{2}{h}}\right), \quad \forall k>0 \text { as } r \rightarrow 0 .
$$

Similarly to (3.1),

$$
r^{2} \int_{B_{r}}|x|^{-2}|w|^{2} \mathrm{~d} x=r^{2} O\left(r^{k-\frac{2}{h}-2}\right)=O\left(r^{k-\frac{2}{h}}\right), \quad \forall k>0 \text { as } r \rightarrow 0 .
$$

Since $1-c<0$, then we have

$$
\begin{aligned}
\int_{B_{r}}|w|^{2} \mathrm{~d} x+(1-c) r^{2} \int_{B_{r}}|x|^{-2}|w|^{2} \mathrm{~d} x & \geq \int_{B_{r}}|w|^{2} \mathrm{~d} x+(1-c) \int_{B_{r}}|w|^{2} \mathrm{~d} x \\
& =(2-c) \int_{B_{r}}|w|^{2} \mathrm{~d} x .
\end{aligned}
$$

Choosing $1<c<2$, we have (3.3) by (3.4) and (3.6).

Now we show that if $x_{0}$ is a zero of infinite order for $u$ which is a solution of system (1.1), then $x_{0}$ is a zero of "exponential" order. Without loss of generality, we suppose $x_{0}=0$.

By Remark 2.1 and Lemma 3.1, we have:

Theorem 3.1. Suppose that $u \in H_{\mathrm{loc}}^{2}\left(\mathbb{R}^{3}\right)$, and satisfies (1.2). Then

$$
\int_{B_{r}}|u|^{2} \mathrm{~d} x=O\left(\mathrm{e}^{-r^{-1}}\right), \quad \text { as } r \rightarrow 0
$$

Proof. Suppose $u \in H_{\mathrm{loc}}^{2}\left(\mathbb{R}^{3}\right)$ is a solution (1.1), and satisfies (1.2). We can obtain that for all $|\alpha| \leq 2$

$$
\int_{B_{r}}\left|D^{\alpha} u\right|^{2} \mathrm{~d} x=O\left(r^{k}\right), \quad \forall k>0 \quad \text { as } r \rightarrow 0 .
$$

Assume that $h$ satisfies the conditions of Lemma 2.1. Choose $k$ sufficiently larger than $\frac{h}{2}$ and a cut-off function $\tilde{\chi} \in C_{c}^{\infty}\left(\mathbb{R}^{3}\right)$ such that

$$
\begin{cases}\tilde{\chi}(x)=1 & \text { as }|x|<r \\ \tilde{\chi}(x)=0 & \text { as }|x|>2 r\end{cases}
$$

It is easy to check that $\tilde{\chi}$ satisfies

$$
\left|D^{\alpha} \widetilde{\chi}(x)\right| \leq C r^{-|\alpha|}
$$

$\tilde{\chi} u \in H^{2}$ has a compact support, then by Remark 2.1, (2.18) also holds for $\tilde{\chi} u$. Thus by Corollary 3.1, we have

$$
\int \tilde{\chi}|x|^{-\frac{2}{h}}|u|^{2} \mathrm{~d} x+\left.\left.h^{2} r^{2} \int|| x\right|^{-\frac{1}{h}} D \widetilde{\chi} u\right|^{2} \mathrm{~d} x \leq C r^{4} \int|x|^{-\frac{2}{h}}|L(\widetilde{\chi} u)|^{2} \mathrm{~d} x .
$$


Noting that

$$
\begin{aligned}
0 & =\operatorname{div}\left(\mu\left(\nabla u+(\nabla u)^{\top}\right)\right)+\nabla(\lambda \operatorname{div} u)+V u \\
& =L u+\left(\nabla u+(\nabla u)^{\top}\right) \nabla \mu+(\operatorname{div} u) \nabla \lambda+V u .
\end{aligned}
$$

It means

$$
\begin{aligned}
\int \tilde{\chi}|x|^{-\frac{2}{h}}|u|^{2} \mathrm{~d} x+ & \left.\left.h^{2} r^{2} \int|| x\right|^{-\frac{1}{h}} D \tilde{\chi} u\right|^{2} \mathrm{~d} x \leq C r^{4} \int|x|^{-\frac{2}{h}}|L(\widetilde{\chi} u)|^{2} \mathrm{~d} x \\
\leq & C r^{4} \int \tilde{\chi}|x|^{-\frac{2}{h}}|L u|^{2}+|x|^{-\frac{2}{h}}|[L, \tilde{\chi}] u|^{2} \mathrm{~d} x \\
\leq & C r^{4}\left(\int_{|x|<r}|x|^{-\frac{2}{h}}|D u|^{2} \mathrm{~d} x+\int_{|x|<r}|x|^{-\frac{2}{h}}|V u|^{2} \mathrm{~d} x\right. \\
& \left.+\int_{r<|x|<2 r}|x|^{-\frac{2}{h}}|u|^{2} \mathrm{~d} x+\int_{r<|x|<2 r}|x|^{-\frac{2}{h}}|D u|^{2} \mathrm{~d} x\right) .
\end{aligned}
$$

Recall that $V \in L^{\infty}(\Omega)$, we have

$$
\begin{aligned}
\int \tilde{\chi}|x|^{-\frac{2}{h}}|u|^{2} \mathrm{~d} x+\left.\left.h^{2} r^{2} \int|| x\right|^{-\frac{1}{h}} D \tilde{\chi} u\right|^{2} \mathrm{~d} x & \leq C_{0} r^{4}\left(\int_{|x|<r}|x|^{-\frac{2}{h}}|D u|^{2} \mathrm{~d} x+\int_{|x|<r}|x|^{-\frac{2}{h}}|u|^{2} \mathrm{~d} x\right. \\
& \left.+\int_{r<|x|<2 r}|x|^{-\frac{2}{h}}|u|^{2} \mathrm{~d} x+\int_{r<|x|<2 r}|x|^{-\frac{2}{h}}|D u|^{2} \mathrm{~d} x\right) .
\end{aligned}
$$

After some calculations, we have

$$
\begin{aligned}
\left(1-C_{0} r^{4}\right) \int_{|x|<r}|x|^{-\frac{2}{h}}|u|^{2} \mathrm{~d} x+\left.\left.\left(h^{2} r^{2}-C_{0} r^{4}\right) \int|| x\right|^{-\frac{1}{h}} D \tilde{\chi} u\right|^{2} \mathrm{~d} x & \\
& \leq C_{0} r^{4}\left(\int_{r<|x|<2 r}|x|^{-\frac{2}{h}}|u|^{2} \mathrm{~d} x+\int_{r<|x|<2 r}|x|^{-\frac{2}{h}}|D u|^{2} \mathrm{~d} x\right)
\end{aligned}
$$

Noting $r$ is sufficiently small, as long as $0 \ll r \ll h \ll 1$, we have

$$
\begin{aligned}
\left.\left.\left(h^{2} r^{2}-C_{0} r^{4}\right) \int|| x\right|^{-\frac{1}{h}} D \tilde{\chi} u\right|^{2} \mathrm{~d} x \geq\left.\left. r^{4} \int|| x\right|^{-\frac{1}{h}} D \tilde{\chi} u\right|^{2} \mathrm{~d} x & \\
& \geq r^{4} \int_{|x|<r}|x|^{-\frac{2}{h}}|D u|^{2} \mathrm{~d} x-r^{4} \int_{r<|x|<2 r}|x|^{-\frac{2}{h}}|u|^{2} \mathrm{~d} x
\end{aligned}
$$

Summing up, we have

$$
\int_{|x|<r}|x|^{-\frac{2}{h}}|u|^{2} \mathrm{~d} x \leq C r^{4} \int_{|x|>r}|x|^{-\frac{2}{h}}|u|^{2} \mathrm{~d} x+C r^{4} \int_{|x|>r}|x|^{-\frac{2}{h}}|D u|^{2} \mathrm{~d} x .
$$

That is

$$
\left(\frac{r}{2}\right)^{-\frac{2}{h}} \int_{|x|<r}|u|^{2} \mathrm{~d} x \leq C r^{-\frac{2}{h}+4} \int_{|x|>r}|u|^{2} \mathrm{~d} x+C r^{-\frac{2}{h}+4} \int_{|x|>r}|D u|^{2} \mathrm{~d} x
$$


It implies

$$
\int_{B_{r}}|u|^{2} \mathrm{~d} x \leq C r^{-2} 2^{-\frac{2}{h}}\|u\|_{H^{1}}^{2} .
$$

Choosing $h=\left(1+C_{0}\right) r$, we note that $h$ has to satisfy $h^{-1} \in A=\{m+2, m \in \mathbb{N}\}$. Then $\left[\left(1+C_{0}\right) r\right]^{-1}$ must be in $A$. But but one can check without difficulty that (3.15) is available for all $r>0$. Thus

$$
\int_{B_{r}}|u|^{2} \mathrm{~d} x=O\left(\mathrm{e}^{-r^{-1}}\right), \quad r \rightarrow 0 .
$$

Then we complete the proof the theorem.

In our previous work [14], we gave a quantitative estimate of unique continuation for a three-dimensional Lamé system with $C^{1}$ coefficients in the form of three spheres inequalities. The property of the non faster than exponential vanishing of nonzero local solutions is also given as an application of the three spheres inequality (1.1).

Theorem 3.2. Assume that $\Omega$ is a bounded domain in $\mathbb{R}^{3}$, the Lamé moduli $\mu, \lambda \in C^{0,1}(\Omega)$ satisfy the strong elliptic conditions. Let $u \in H^{2}(\Omega)$ be a solution to (1.1). If there is a point $x_{0} \in \Omega$ and $k>0$ such that,

$$
\int_{\left|x-x_{0}\right|<r}|u|^{2} \mathrm{~d} x=O\left(\mathrm{e}^{-r^{-k}}\right), \quad \text { as } r \rightarrow 0^{+} .
$$

Then $u \equiv 0$, in $\Omega$.

Then the SUCP follows immediately by Theorems 3.1 and 3.2 .

Acknowledgements. The author wants to show his great gratitude to Professor Hongwei Lou and Professor Xu Zhang for many helpful guidance and suggestions.

\section{REFERENCES}

[1] G. Alessandrini and A. Morassi, Strong unique continuation for the Lamé system of elasticity. Comm. P. D. E. 26 (2001) $1787-1810$.

[2] D.D. Ang, M. Ikehata, D.D. Trong and M. Yamamoto, Unique continuation for a stationary isotropic Lamé system with varaiable coefficients. Comm. P. D. E. 23 (1998) 371-385.

[3] B. Dehman and L. Robbiano, La propriété du prolongement unique pour un système elliptique : le système de Lamé. J. Math. Pures Appl. 72 (1993) 475-492.

[4] M. Eller, Carleman estimates for some elliptic systems. J. Phys. Conference Series 124 (2008) 012023.

[5] L. Escauriaza, Unique continuation for the system of elasticity in the plan. Proc. Amer. Math. Soc. 134 (2005) $2015-2018$.

[6] C.E. Kenig, J. Sjöstrand and G. Uhlmann, The Calderón problem with partial data. Ann. Math. 165 (2007) 567-591.

[7] C.-L. Lin, G. Nakamura and J.-N. Wang, Optimal three-ball inequalities and quantitative uniqueness for the Lamé system with Lipschitz coefficients. arXiv:0901.4638 (2009).

[8] C.-L. Lin and J.-N. Wang, Strong unique continuation for the Lamé system with Lipschitz coefficients. Math. Ann. 331 (2005) $611-629$.

[9] A. Martinez, An introduction to semiclassical and microlocal analysis. Springer-Verlag (2002).

[10] R. Regbaoui, Strong uniqueness for second order differential operators J. Differ. Equ. 141 (1997) 201-217.

[11] M. Salo and L. Tzou, Carleman estimates and inverse problems for Dirac operators. Math. Ann. 344 (2009) 161-184.

[12] N. Weck, Außnraumaufgaben in der Theorie stationärer Schwingungen inhomogener elasticher Körper. Math. Z. 111 (1969) 387-398.

[13] N. Weck, Unique continuation for systems with Lamé principal part. Math. Methods Appl. Sci. 24 (2001) 595-605.

[14] H. Yu, Three spheres inequalities and unique continuation for a three-dimensional Lamé system of elasticity with $C^{1}$ coeffients. arXiv:0811.1262 (2008). 